\title{
Emission spectrum of quasiresonant laterally coupled quantum dots
}

\author{
Miquel Royo, Juan I. Climente, and Josep Planelles* \\ Departament de Química Física i Analítica, Universitat Jaume I, E-12080, Castelló, Spain
}

(Received 28 June 2011; revised manuscript received 29 November 2011; published 21 December 2011)

\begin{abstract}
We calculate the emission spectrum of neutral and charged excitons in a pair of laterally coupled InGaAs quantum dots with nearly degenerate energy levels. As the interdot distance decreases, a number of changes take place in the emission spectrum which can be used as indications of molecular coupling. These signatures ensue from the stronger tunnel coupling of trions as compared to that of neutral excitons.
\end{abstract}

DOI: 10.1103/PhysRevB.84.235312

PACS number(s): 73.21.La, 78.67.Hc, 78.55.Cr, 71.35.-y

\section{INTRODUCTION}

There is current interest in developing quantum dot molecules (QDMs) for quantum-information processing. ${ }^{1,2}$ Great progress in this direction has been made using vertical QDMs fabricated with epitaxial growth techniques. These structures are formed by pairs of vertically stacked QDs with a thin barrier in between. Because the constituent QDs are generally asymmetric, electric fields applied along the coupling direction are used to control the charge and the tunnel coupling strength. ${ }^{3-6}$

In the last few years, development of QDMs formed by laterally coupled QD pairs has also been pursued. ${ }^{7-12}$ In these structures, the QD pair is formed inside the same epitaxial layers. This architecture is expected to offer some advantages over vertical QDMs. ${ }^{13,14}$ In particular, independent control of QD charging and tunneling strength should be possible through the application of vertical and lateral electric fields, respectively. Also, simultaneous coupling of multiple QDMs, which is a requirement for the scaling of qubit operations, should be feasible through the implementation of individual electric gates for each QDM. On the other hand, lateral QDMs present their own obstacles. For example, the distance between the centers of the QDs is much larger than in vertical structures, which implies weaker tunneling. The realization and control of coherent molecular tunneling is a prerequisite for spin manipulation through exchange interactions, ${ }^{1}$ as recently achieved in vertical QDMs. ${ }^{15}$ Despite recent advances in the electrical engineering of lateral QDMs, which include the demonstration of Coulomb blockade charging ${ }^{16-18}$ and tuning of optical resonances,${ }^{19}$ the application of lateral electric fields producing clear spectroscopic signatures of molecular tunnel coupling remains a challenge. Thus, the research on lateral QDMs is still focused on this preliminary step.

Understanding the differences between the physics of lateral QDMs and the better known case of vertical QDMs will contribute to determining the conditions for optimal device performance, be it vertical, lateral, or hybrid. ${ }^{20}$ Many of the differences arise from the distinct synthetic routes, which lead to characteristic structural and compositional profiles. ${ }^{19,21}$ For example, in lateral QDMs it has been shown that the weaker quantum confinement of the QDs along the coupling direction leads to an enhanced role of the excited orbitals within the QDs. ${ }^{22,23}$ Also, the nonradiative relaxation dynamics of lateral InGaAs QDMs has been shown to follow a singular path. ${ }^{19,21}$

More recently, Peng and Bester used atomistic modeling to investigate the electronic structure and emission spectrum of different excitonic complexes in lateral QDMs subject to external electric fields. ${ }^{24}$ They identified spectroscopic signatures induced by the electric field which should reveal the presence of tunnel coupling under experimentally feasible conditions.

In this paper, we study the optical resonances of neutral, negatively charged, and positively charged excitons in lateral InGaAs QDMs. Because electric control of these structures is not fully mature, we focus on the case of zero electric field and vary the interdot distance instead. We consider slightly asymmetric QDs, as often obtained with current growth techniques, ${ }^{7,9,16,17,21}$ and show that one can identify signatures of tunnel coupling even in the zero-field photoluminescence spectrum. This should be useful in distinguishing uncoupled QD pairs from coupled QDMs. We also show that tunnel coupling of trions is stronger than that of neutral excitons. The convenience of using trions instead of neutral excitons for quantum-information protocols is discussed.

\section{THEORETICAL MODEL}

Our Hamiltonian for excitons and trions can be written in the second quantization as

$$
\begin{aligned}
\hat{H}= & \sum_{i} E_{i}^{e} e_{i}^{+} e_{i}+\sum_{p} E_{p}^{h} h_{p}^{+} h_{p} \\
& +\frac{1}{2} \sum_{i j k l}\langle i j|V| k l\rangle e_{i}^{+} e_{j}^{+} e_{k} e_{l} \\
& +\frac{1}{2} \sum_{p q r s}\langle p q|V| r s\rangle h_{p}^{+} h_{q}^{+} h_{r} h_{s} \\
& +\sum_{i j p q}\langle i p|V| q j\rangle e_{i}^{+} h_{p}^{+} h_{q} e_{j},
\end{aligned}
$$

where $E_{i}^{e}\left(E_{p}^{h}\right)$ is the electron (hole) energy in the singleparticle state $|i\rangle(|p\rangle), e_{i}^{+} / e_{i}\left(h_{p}^{+} / h_{p}\right)$ is the electron (hole) creation or annihilation operator, i.e., $\left(e_{i}^{+} e_{j}^{+} \ldots\right)\left(h_{p}^{+} h_{q}^{+} \ldots\right)$ $\left|0_{h}\right\rangle\left|0_{e}\right\rangle=|p q \ldots\rangle|i j \ldots\rangle$, and $\langle i j|V| k l\rangle,\langle p q|V| r s\rangle$, and $\langle i p|V| q j\rangle$ are the electron-electron, hole-hole, and electronhole Coulomb matrix elements, respectively. The sums in Eq. (1) run over spin orbitals, so both direct and exchange Coulomb terms are considered. The electron-hole exchange term is, however, neglected. This is a fine effect whose only influence on the bright excitonic states at zero magnetic field is a splitting of tens of $\mu \mathrm{eV}$. Consequences of anisotropic 


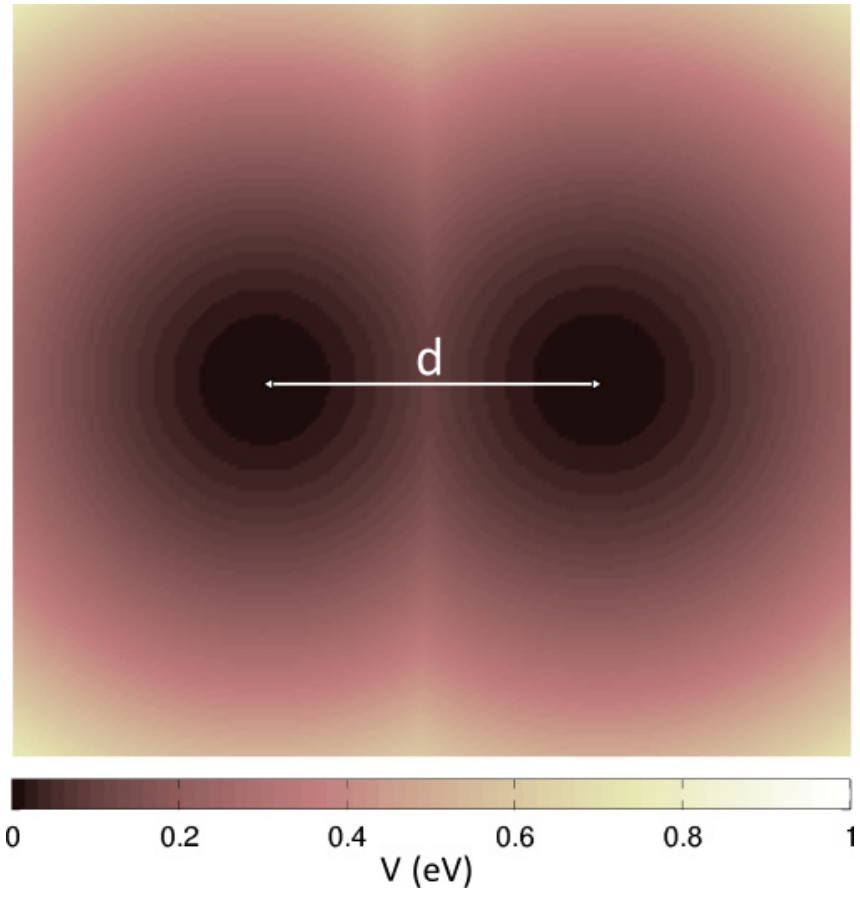

FIG. 1. (Color online) Contour plot of the confinement potential for slightly asymmetric, laterally coupled QDs with an interdot distance $d=40 \mathrm{~nm}$.

electron-hole exchange on the polarization of the emitted light are not relevant to the purpose of this study.

To calculate the single-particle states, we use a twodimensional effective mass Hamiltonian for electrons and heavy holes:

$$
\hat{H}_{i}=\frac{1}{2 m_{i}^{*}}\left(p_{x}^{2}+p_{y}^{2}\right)+V_{i}(x, y),
$$

where $i=e, h$ is the index denoting electrons or holes, $m^{*}$ is the effective mass, $p_{\alpha}=-i \hbar \nabla_{\alpha}$ the momentum operator, and $V(x, y)$ the quantum confinement potential. For a pair of QDs whose centers are separated by a distance $d, V(x, y)$ is defined with intersecting parabolic potentials:

$$
\begin{aligned}
V_{i}(x, y)= & \frac{1}{2} m_{i}^{*}\left(\operatorname { m i n } \left\{\omega_{i, L}^{2}\left[\left(x+\frac{d}{2}\right)^{2}+y^{2}\right],\right.\right. \\
& \left.\left.\omega_{i, R}^{2}\left[\left(x-\frac{d}{2}\right)^{2}+y^{2}\right]\right\}\right) .
\end{aligned}
$$

Here $\omega_{i, L}\left(\omega_{i, R}\right)$ is the confinement frequency in the left (right) QD. A contour plot of this potential profile for a slightly asymmetric QDM is shown in Fig. 1. Similar models were previously adopted to study laterally coupled QDs. ${ }^{25,26}$ The use of a single-band, heavy hole description for the valence band states in single QD has been generally found to be accurate, as the three-dimensional confinement pushes light holes far in energy. ${ }^{27}$ In vertical QDMs with large interdot distances, heavy-hole-light-hole coupling can become important, ${ }^{28,29}$ but this does not seem to be the case for lateral molecules. ${ }^{30}$ Due to its continuum nature, the effective mass model misses microscopic and interface effects which are captured by atomistic models. However, effective mass models have proved extremely useful in the study of vertical
QDMs, where they correctly describe most relevant aspects connected with molecular tunneling. ${ }^{2,5,28}$ One can then expect these models to be equally valid for the study of lateral QDMs.

Hamiltonian (2) is integrated numerically using a finitedifferences scheme. The resulting electron and hole states are plugged into Hamiltonian (1), together with the Coulomb matrix elements, which we calculate using an adaptive Monte Carlo routine. ${ }^{31}$ The Hamiltonian is then solved using a configuration interaction routine. ${ }^{32}$ In this way, we obtain an accurate description of the ground and excited states of the QDM system. To simulate their emission spectrum, we use the dipole approximation and Fermi's golden rule. ${ }^{27}$ The recombination probability from an initial few-body state $|i\rangle$ to a final state $|f\rangle$ with one less electron-hole pair, at an emission frequency $\omega$ is then given by

$$
\tau_{f \leftarrow i}^{-1}(\omega) \propto|\langle f|\hat{\mathcal{P}}| i\rangle|^{2} \Gamma\left(E^{i}-E^{f}-\hbar \omega\right) p_{i}(T),
$$

where $\hat{\mathcal{P}}$ is the polarization operator, ${ }^{33} E^{s}$ is the energy of the state $|s\rangle$, and $\Gamma(E)$ is a Lorentzian curve centered at energy $E$, which simulates the intrinsic bandwidth of the transition. $p_{i}(T)$ is the thermal population distribution function for the initial state at temperature $T$. We shall consider two possible dynamics for the emission process: (i) Emission takes place from all the excited states, which are populated through nonresonant excitation, prior to their thermal relaxation. We simulate this adopting the same occupation probability for all the initial states, $p_{i}(T)=1$. (ii) Recombination takes place once the thermal equilibrium is reached. In this case we assume a Boltzmann distribution $p_{i}(T)=Z \frac{g_{i}}{g_{0}} e^{-\frac{\Delta E_{i}}{k T}}$. Here $g_{i}\left(g_{0}\right)$ is the degeneracy factor of the state $|i\rangle$ (ground state), $\Delta E_{i}$ the energy difference between $|i\rangle$ and the ground state, $k$ the Boltzmann constant, and $Z$ the normalization constant.

In our calculations we consider laterally coupled InGaAs/GaAs QDs. We shall use parameters which, for long interdot distances, fit the emission spectrum observed in single QDs fabricated upon GaAs nanohole templates. ${ }^{10,17}$ Effective masses are $m_{e}^{*}=0.0324$ and $m_{h}^{*}=0.435$, as corresponding to $\operatorname{In}_{0.75} \mathrm{Ga}_{0.25} \mathrm{As}$, and the dielectric constant $\epsilon=12.3 .^{34}$ The electron confinement frequencies are $\hbar \omega_{e, L}=35 \mathrm{meV}$ and $\hbar \omega_{e, R}=34 \mathrm{meV}$, so that the right QD is slightly bigger than the left QD. Note that small asymmetries can be found in some real samples. ${ }^{16,17}$ Hole confinement frequencies are taken such that $l_{h}=0.59 l_{e}$, where $l_{i}$ is the characteristic length, $l_{i}=\sqrt{\hbar / m_{i}^{*} \omega_{i}}$. This means that holes are more confined than electrons, and their tunneling will be negligible. Similar $l_{h} / l_{e}$ ratios have been estimated in recent experiments with InGaAs QDs. ${ }^{35}$ The bandwidth of the Lorentzian function in our spectral simulations is $0.05 \mathrm{meV}$. For the many-body calculation, we project Hamiltonian (1) onto all the singleparticle configurations which can be obtained by combining the 24 lowest-energy electron and hole spin orbitals. For excitons (trions), this gives rise to 576 (6624) configurations. The origin of electron (hole) single-particle energies is taken at the edge of the conduction (valence) band, disregarding the $z$-confinement energy, which is constant for all the states. 


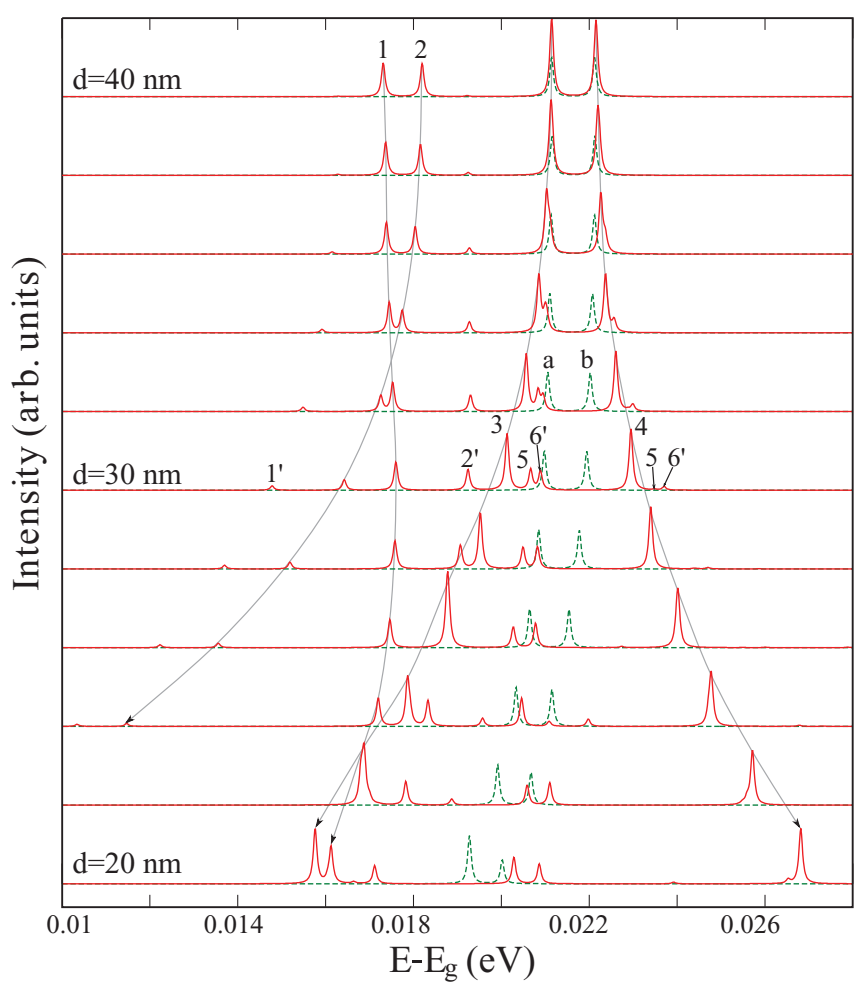

FIG. 2. (Color online) Emission spectrum of negative trions (solid red lines) and neutral excitons (dashed green lines) for different interdot distances. Grey lines are a guide to the eyes. See text for the meaning of labels.

\section{RESULTS AND DISCUSSION}

\section{A. Exciton and negative trion}

Figure 2 shows the emission spectrum of the QDM as a function of the interdot distance $d$, from $d=40 \mathrm{~nm}$ (isolated QDs) to $d=20 \mathrm{~nm}$ (strongly coupled QDs). Solid red and dashed green lines are used for negative trions $\left(X^{-}\right)$and neutral excitons $\left(X^{0}\right)$, respectively. Here we assume an outof-equilibrium system. In this way, we simulate optically active transitions from ground and excited states of both QDs, as normally observed in the photoluminescence spectra of vertical ${ }^{4-6}$ and lateral ${ }^{16-18}$ QDMs. The most remarkable result of Fig. 2 is that the $X^{-}$and $X^{0}$ resonances follow very different evolutions as the QDs are brought closer together. To understand the different behavior, we need to analyze each excitonic complex in detail.

In the limit of the uncoupled QDM, $d=40 \mathrm{~nm}, X^{0}$ shows two resonances of similar intensity split by $\sim 1 \mathrm{meV}$. As $d$ is reduced, the two resonances are slowly redshifted, but the energy splitting remains approximately constant. This behavior can be understood from the exciton states, plotted in Fig. 3. The emission resonances correspond to the two lowest $X^{0}$ states. At $d=40 \mathrm{~nm}$, these are the direct excitons, with the electron-hole pair in the big (low-energy resonance) and small (high-energy resonance) QD, see right-side insets in the figure. Indirect excitons (electron and hole in opposite QDs) are much higher in energy and optically dark because the electron-hole overlap is negligible. As the interdot distance decreases, tunnel coupling allows electrons and holes to start hybridizing and forming incipient molecular orbitals, even if the QDs are not

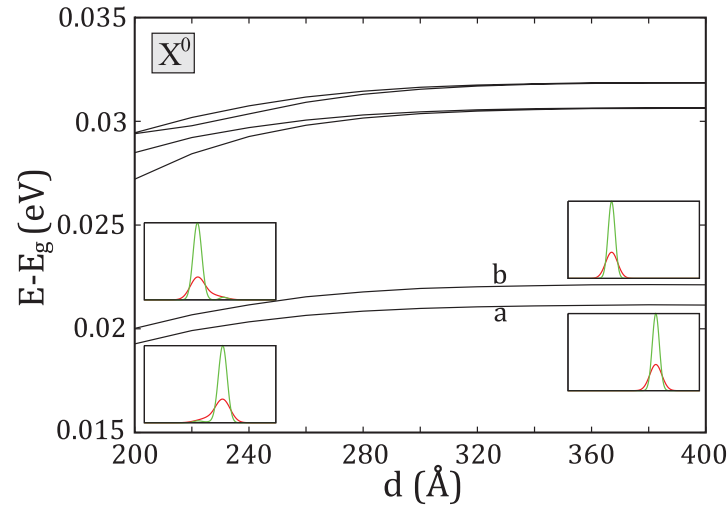

FIG. 3. (Color online) Energy of the lowest exciton states as a function of the interdot distance. The insets show the charge density of the electron (red or dark gray line) and hole (green or light gray line) within the exciton complex for the optically active states at $d=40 \mathrm{~nm}$ (right) and $d=20 \mathrm{~nm}$ (left).

exactly resonant. The lowest $X^{0}$ state corresponds to bonding (nodeless) electron and hole orbitals, so it is redshifted. The first excited state corresponds to a bonding electron but an antibonding hole orbital (note the presence of a node in the green line of the upper-left inset). Yet, this makes little difference because hole tunneling is negligible. As a result, the two states are redshifted by a similar amount.

The $X^{0}$ emission spectrum shown in Fig. 2 differs from previous calculations for asymmetric lateral QDMs, where the two lowest $X^{0}$ states were at some point blueshifted by Coulomb coupling. ${ }^{7}$ This is due to the different confining potentials in the models. In Ref. 7, the interdot barrier was a steplike potential. Instead, we use a parabolic potential such that, with decreasing $d$, not only are the QDs closer but also the barrier is lower. For this reason, tunnel-coupling effects rapidly overcome Coulomb coupling in our system. Real QDMs are lens-shaped QDs with some degree of lateral contact. ${ }^{10}$ As the dots are brought closer, the height in the contact region increases. We then believe our model is more appropriate.

Next we investigate the $X^{-}$spectrum. As can be seen in Fig. 2, at $d=40 \mathrm{~nm}$ there are four resonances associated with $X^{-}$states. Two resonances, (1) and (2), are a few meV below those of $X^{0}$, while two are degenerate with the $X^{0}$ ones. As $d$ is reduced, several features show up which differ from the simple behavior of neutral excitons: (i) the number of resonances increases (up to 10); (ii) the two low-energy resonances invert their ordering; and (iii) the degeneracy between the high-energy $X^{-}$and the $X^{0}$ resonances is lifted. Significantly, most of this occurs even before the neutral exciton starts experiencing tunnel coupling (see, e.g., $d=34 \mathrm{~nm})$.

To understand the $X^{-}$emission spectrum, we study the energy levels of the initial $\left(X^{-}\right)$and final $\left(1 e^{-}\right)$states, which are plotted in Fig. 4. The electron, Fig. 4(b), displays a simple dissociation spectrum typical of diatomic heteronuclear molecules. At $d=40 \mathrm{~nm}$ the electron is in one of the two QDs (see right insets). With decreasing $d$, it forms bonding and antibonding (noded) molecular orbitals (see left insets). As for the trion, Fig. 4(a), the two lowest-energy states correspond to the so-called direct trion states. Their behavior is the same as 


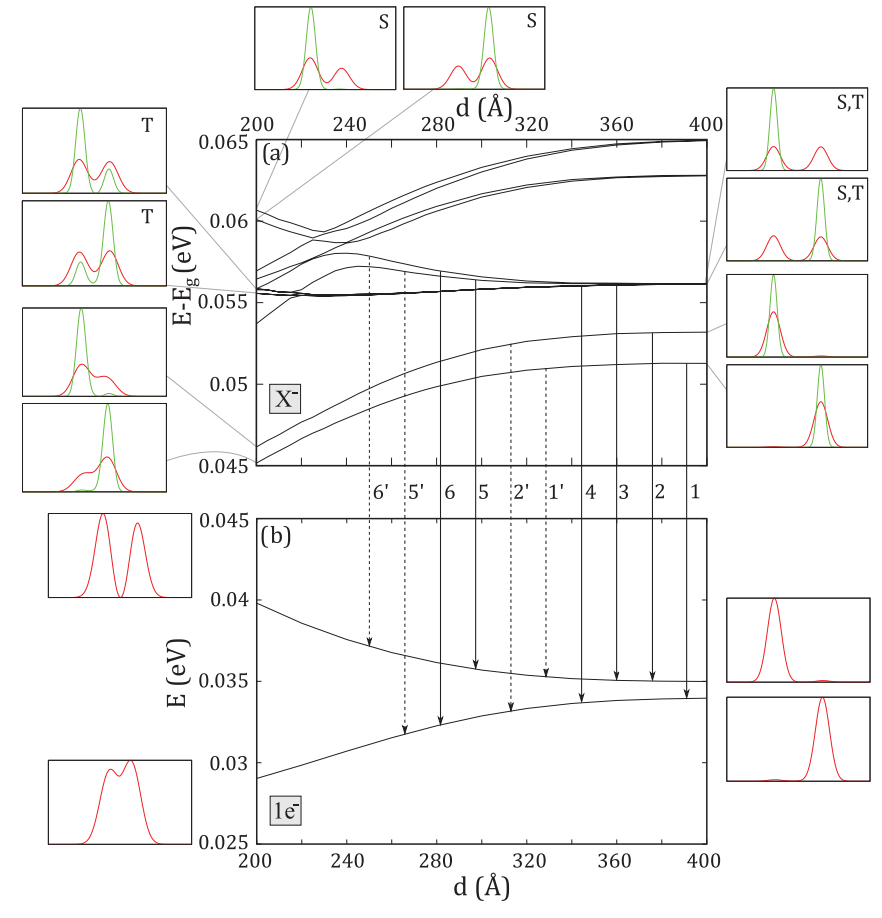

FIG. 4. (Color online) Energy of the lowest negative trion states (a) and electron states (b) as a function of the interdot distance. The lateral insets show the charge density of the electron (red or dark gray line) and hole (green or light gray line) at $d=40 \mathrm{~nm}$ (right) and $d=20 \mathrm{~nm}$ (left). Vertical arrows indicate the transitions observed in Fig. 2.

that of $X^{0}$. Namely, at $d=40 \mathrm{~nm}$ all the carriers are localized inside one of the QDs, and they are redshifted with decreasing $d$ owing to electron hybridization (see insets). As indicated by the vertical arrows in Fig. 4, resonance 1 (2) in Fig. 2 originates in the transition from the direct $X^{-}$state in the right (left) QD to the electron state in the same QD. The energy splitting between the two initial states is larger than that between the final states. As a result, transition (1) is lower in energy. However, as $d$ decreases the situation reverses. Transition (1) involves bonding initial and final states, whose energetic stabilizations due to tunnel coupling compensate each other. As a result, the energy of the transition is little sensitive to the coupling (see Fig. 2). In contrast, transition (2) involves a bonding initial state but an antibonding final state, so its energy rapidly decreases. This is also seen in Fig. 2 and leads to a reversal of resonances (1) and (2) at $d \sim 32 \mathrm{~nm}$. What is more, because of the QDM asymmetry, transitions between bonding and antibonding states are not symmetry forbidden. Then, when electron hybridization takes place, new transitions start developing, $\left(1^{\prime}\right)$ and $\left(2^{\prime}\right)$.

Resonances (3)-(6) in the $X^{-}$spectrum originate in a set of excited trion states with energy $\sim 0.055 \mathrm{eV}$ at $d=40 \mathrm{~nm}$. These are the so-called indirect trions, where one electron sits in a QD and the remaining electron-hole pair sits in the opposite QD (see insets in Fig. 4). When the electron-hole pair recombines, the electron in the opposite QD remains. At $d=40 \mathrm{~nm}$, the Coulomb interaction between the two QDs is so weak that the indirect trion resonances have the same energy as those of $X^{0}$. With decreasing $d$, however, the behavior of indirect trions becomes noticeably different from that of neutral excitons. The indirect-trion states split into two groups. One group is formed by states where the two electrons form a singlet spin configuration. These states increase in energy and eventually anticross with higher states. This destabilization is mainly caused by the gradual enhancement of the antibonding character. In contrast, the group of states with electrons forming a triplet remains almost insensitive to tunnel coupling because of the spin blockade. This effect is analogous to that observed in vertical QDMs under electric fields, where the Pauli exclusion principle prevents triplet states from tunneling. ${ }^{4}$ The characteristic evolution of indirect trions vs $d$ is responsible for the lifting of the degeneracy with $X^{0}$ resonances observed in Fig. 2. Last, as in the case of direct trions, the QDM asymmetry enables transitions from indirect trions to either bonding or antibonding electron states, which gives rise to new resonances when electrons hybridize, $\left(5^{\prime}\right)$ and $\left(6^{\prime}\right)$.

The different evolution of $X^{-}$and $X^{0}$ resonances in the emission spectrum of QDMs can be used to elucidate the presence of molecular coupling. The appearance of additional trion resonances should be a clear signature, but they may be too weak for typical interdot distances $(d=30-40 \mathrm{~nm}$ in Ref. 10). A more evident signature should be the splitting between $X^{0}$ and indirect $X^{-}$resonances, which involves intense optical resonances and is visible for long interdot distances $(d \sim 36 \mathrm{~nm}$ in Fig. 2). Finally, the reversal of resonances (1) and (2) may also be noticed in experiments. For $d<34 \mathrm{~nm}$, this implies that trion emission from the bigger QD occurs at higher energy than that from the smaller QD, contrary to the ordering of $X^{0}$ resonances. As a matter of fact,

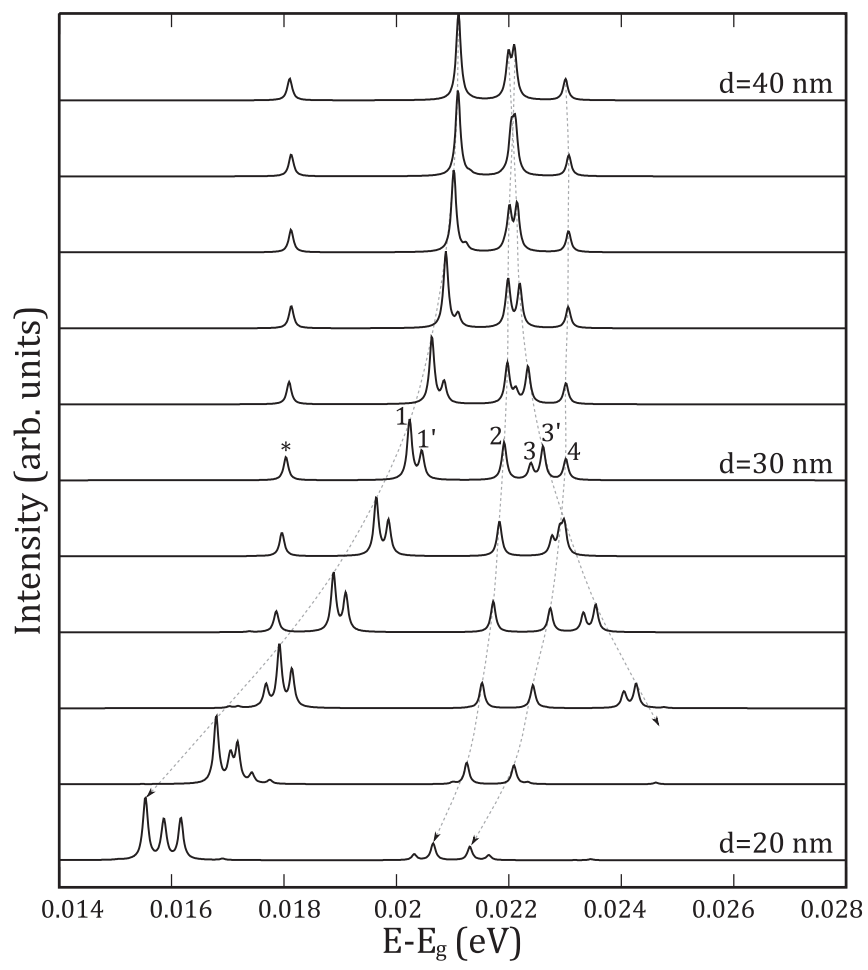

FIG. 5. Emission spectrum of positive trions for different interdot distances. Dashed lines are a guide to the eyes. 
this anomalous spectral ordering has been recently found in photoluminescence measurements of lateral QDMs. ${ }^{17}$

\section{B. Positive trion}

We next study the emission spectrum of the positive trion $\left(X^{+}\right)$. In this case, assuming equal population for all the initial states would lead to a complex spectrum with many resonances involving highly excited initial and final states. Since we are interested in the main transitions only, the emission spectrum plotted in Fig. 5 has been computed assuming thermal equilibrium at $T=80 \mathrm{~K}$. At this temperature, the $X^{+}$states with significant population are essentially the same as previously studied for $X^{-}$. The only remaining signature of transitions between excited states is the low-energy resonance labeled with an asterisk in Fig. 5, which will not be studied here.

Comparing Figs. 5 and 2 one can see that the $X^{+}$emission pattern differs considerably from the $X^{-}$one. To analyze the emission spectrum, we represent in Fig. 6 the initial $\left(X^{+}\right)$and final $\left(1 h^{+}\right)$states as a function of the interdot distance. The first important difference with respect to $X^{-}$can be seen in the final state, Fig. 6(b). Unlike for electrons, the two lowest hole states remain almost unaltered throughout the entire range of $d$, due to the negligible tunneling. This means that the evolution of the $X^{+}$emission spectrum can be entirely interpreted from the initial $X^{+}$states. The second important difference is in the $X^{+}$states, plotted in Fig. 6(a). There we find two direct and two indirect states, as in the $X^{-}$case, but now their energy

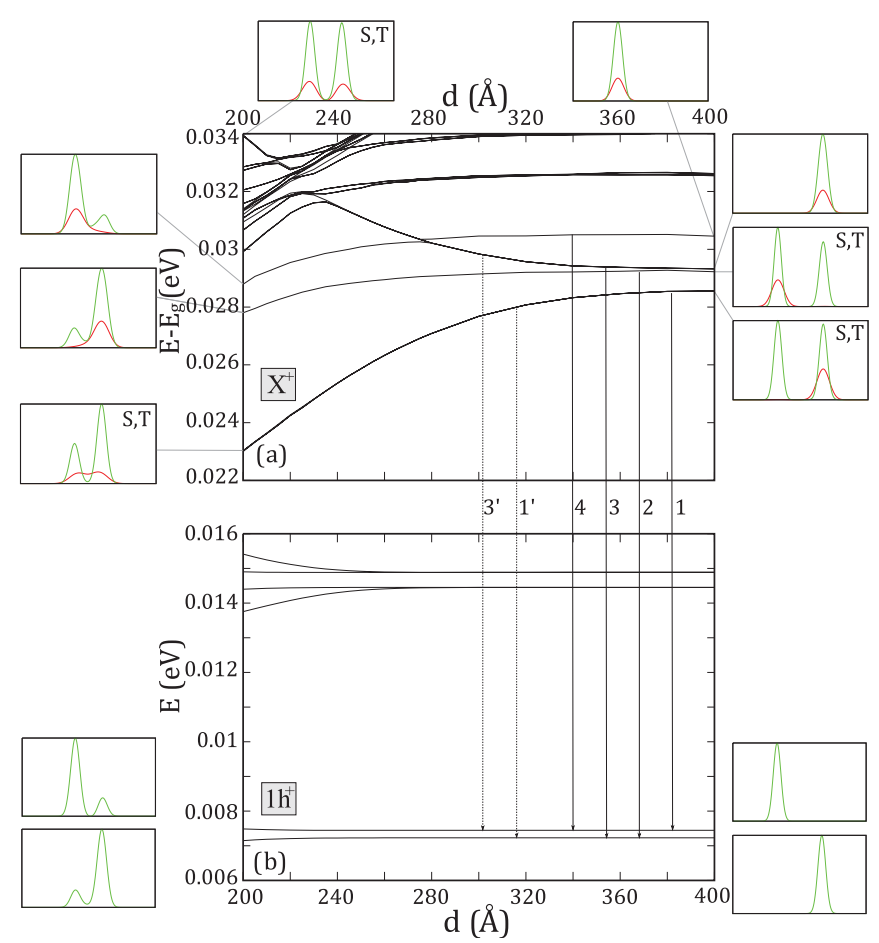

FIG. 6. (Color online) Energy of the lowest positive trion states (a) and hole states (b) as a function of the interdot distance. The lateral insets show the charge density of the electron (red or dark gray line) and the hole (green or light gray line) at $d=40 \mathrm{~nm}$ (right) and $d=20 \mathrm{~nm}$ (left). Vertical arrows indicate the transitions observed in 5 . ordering is reversed. At $d=40 \mathrm{~nm}$, the two lowest states are indirect trions, with one hole in each QD and the electron in either the left or right QD (see insets). We note that indirect $X^{+}$ground states have also been obtained in three-dimensional atomistic calculations, ${ }^{24}$ and are due to the larger magnitude of the hole-hole Coulomb repulsion as compared to the electronhole attraction, i.e., $\left|V_{h h}\right|>\left|V_{e h}\right|$. The situation is the opposite to that of $X^{-}$, where $\left|V_{e e}\right|<\left|V_{e h}\right|$, which favors direct trion configurations.

The resonances labeled as (2) and (4) in Fig. 5 originate in the recombination of the direct trions. As the interdot distance is reduced, they redshift slowly. The evolution of the indirect trion resonances, (1) and (3), is, however, drastic. The two resonances become rapidly split with decreasing $d$, reflecting the strong splitting of indirect trions in Fig. 6(a). Such a large splitting follows from the strong tunneling of indirect $X^{+}$. Unlike for $X^{0}$, the electron in indirect $X^{+}$can tunnel back and forth without losing the strong on-site Coulomb attraction $V_{e h}$. This clearly favors molecular coupling. ${ }^{36}$ Furthermore, the electron delocalization over the entire QDM enables effective recombination with both holes, leading to the appearance of additional resonances, $\left(1^{\prime}\right)$ and $\left(3^{\prime}\right)$, at relatively long interdot distances (see, e.g., $d=36 \mathrm{~nm}$ ).

\section{Low-temperature spectra}

In this section, we reexamine the emission spectra assuming thermal equilibrium at low temperature. This greatly simplifies the spectrum, because only populated initial states contribute to the spectrum. We consider $T=25 \mathrm{~K}$, which is enough for both QDs to have finite occupation. Figure 7 shows the emission

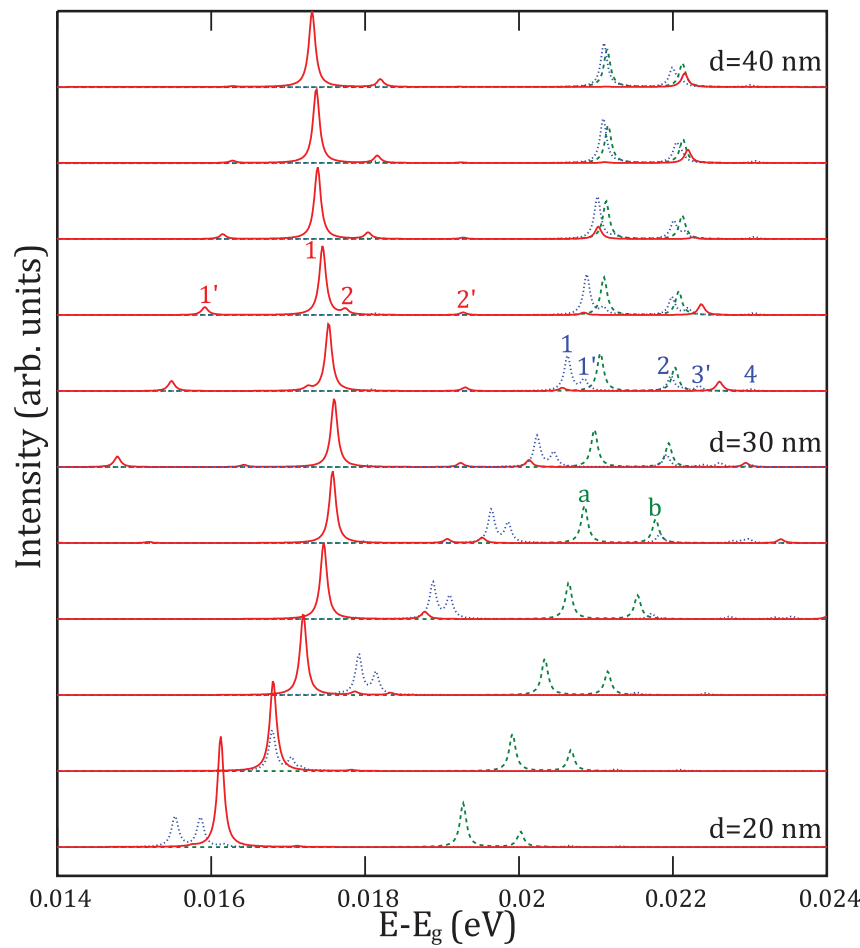

FIG. 7. (Color online) Emission spectrum of negative trions (solid red lines), neutral excitons (dashed green line), and positive trions (dotted blue lines) for different interdot distances. 
spectrum for $X^{-}$(solid red lines), $X^{0}$ (dashed green lines), and $X^{+}$(dotted blue lines). The evolution of $X^{0}$ and $X^{-}\left(X^{+}\right)$ resonances is essentially that of the direct (indirect) species discussed above. Under these conditions, the only signatures of coupling remaining are (i) the appearance of weak $X^{-}$ resonances, solid line peak labeled in red as $\left(1^{\prime}\right)$, and (ii) the presence of strong low-energy $X^{+}$resonances. These transitions arise from the ground (indirect) $X^{+}$state, which soon split into (1) and $\left(1^{\prime}\right)$ and undergo a rapid redshift with decreasing $d$. Thus, at $d=40 \mathrm{~nm}$ we start with a typical spectrum of isolated QDs, where $X^{0}$ and $X^{+}$resonances are close in energy, while $X^{-}$resonances are a few meV below. ${ }^{37,38}$ As $d$ is reduced, the $X^{+}$resonances redshift and approach those of $X^{-}$.

\section{CONCLUSIONS}

We have compared the emission spectrum of $X^{0}, X^{-}$, and $X^{+}$in asymmetric lateral QDMs as a function of the interdot distance. We have shown that tunneling of trions is stronger than that of neutral excitons. This is because the net Coulomb interactions the electron has to overcome in order to tunnel are smaller. As a result, $X^{ \pm}$display signatures of molecular coupling at longer interdot distances than $X^{0}$. The signatures include (i) the appearance of additional optical resonances; (ii) the inversion of the ordering of direct trion resonances corresponding to the big and small QDs; (iii) the lifting of the degeneracy between indirect trion and neutral exciton resonances; and (iv) a pronounced redshift of the direct $X^{+}$resonance, which approaches that of $X^{-}$. We note that signature (ii) has been actually observed in recent photoluminescence experiments. ${ }^{17}$ We have also shown that $X^{-}$and $X^{+}$develop different emission patterns.

These results are particularly valuable in view of recent experimental progress to control the charge of excitonic complexes in QDMs. ${ }^{16-18}$ It has been recently shown that trions are more sensitive than excitons to electric fields, owing to their net electric charge. ${ }^{24}$ This, together with our results, suggests that current attempts to utilize lateral QDMs should focus on trion species rather than neutral excitons. In both positive and negative trions, electron tunneling can be efficient. Yet, positive trions might be less suited because the spin of holes is very sensitive to the in-plane confinement anisotropy. ${ }^{39,40}$ In a lateral QDM, the $X^{+}$ground state contains one hole in each QD. The holes then experience the mutual Coulomb repulsion as a source of anisotropic confinement, which translates into spin mixing. This could hamper the implementation of spin-based quantum-information protocols.

\section{ACKNOWLEDGMENTS}

We thank B. Alén, J. Martínez-Pastor, and G. MuñozMatutano for fruitful discussions. Support from MCINN project CTQ2008-03344, UJI-Bancaixa project P1-1A200903, a Generalitat Valenciana FPI grant (MR), and the Ramon y Cajal program (JIC) is acknowledged. *josep.planelles@uji.es [http://quimicaquantica.uji.es/].

${ }^{1}$ G. Burkard, D. Loss, and D. P. DiVincenzo, Phys. Rev. B 59, 2070 (1999).

${ }^{2}$ M. Bayer, P. Hawrylak, K. Hinzer, S. Fafard, M. Korkusinski, Z. R. Wasilewski, O. Stern, and A. Forchel, Science 291, 451 (2001).

${ }^{3}$ G. Ortner, M. Bayer, Y. Lyanda-Geller, T. L. Reinecke, A. Kress, J. P. Reithmaier, and A. Forchel, Phys. Rev. Lett. 94, 157401 (2005).

${ }^{4}$ H. J. Krenner, M. Sabathil, E. C. Clark, A. Kress, D. Schuh, M. Bichler, G. Abstreiter, and J. J. Finley, Phys. Rev. Lett. 94, 057402 (2005).

${ }^{5}$ H. J. Krenner, E. C. Clark, T. Nakaoka, M. Bichler, C. Scheurer, G. Abstreiter, and J. J. Finley, Phys. Rev. Lett. 97, 076403 (2006).

${ }^{6}$ E. A. Stinaff, M. Schneibner, A. S. Bracker, I. V. Ponomarev, V. L. Korenev, M. E. Ware, M. F. Doty, T. L. Reinecke, and D. Gammon, Science 311, 636 (2006).

${ }^{7}$ M. Baira, L. Sfaxi, L. Bouzaiene, H. Maaref, N. Chauvin, and C. Bru-Chevallier, J. Appl. Phys. 104, 064314 (2008).

${ }^{8}$ L. Wang, A. Rastelli, S. Kiravittaya, P. Atkinson, F. Ding, C. C. Bof Bufon, C. Hermandnstädter, M. Witzany, G. J. Beirne, P. Michler, and O. G. Schmidt, New J. Phys. 10, 045010 (2008).

${ }^{9}$ G. J. Beirne, C. Hermannstadter, L. Wang, A. Rastelli, O. G. Schmidt, and P. Michler, Phys. Rev. Lett. 96, 137401 (2006).

${ }^{10} \mathrm{P}$. Alonso-Gonzalez, J. Martin-Sanchez, Y. Gonzalez, B. Alen, D. Fuster, and L. Gonzalez, Cryst. Growth Des. 9, 2525 (2009).

${ }^{11}$ M. Yamagiwa, T. Mano, T. Kuroda, T. Tateno, K. Sakoda, G. Kido, N. Koguchi, and F. Minami, Appl. Phys. Lett. 89, 113115 (2006).
${ }^{12}$ J. Lee, Z. M. Wang, V. G. Dorogan, Y. I. Mazur, and G. J. Salamo, IEEE Trans. Nanotechnol. 9, 149 (2010).

${ }^{13}$ L. Wang, A. Rastelli, S. Kiravittaya, M. Benyoucef, and O. G. Schmidt, Adv. Mater. 21, 2601 (2009).

${ }^{14}$ V. Krapek, P. Klenowsky, A. Rastelli, O. G. Schmidt, and D. Munzar, J. Phys. Conf. Ser. 245, 012027 (2010).

${ }^{15}$ D. Kim, S. G. Carter, A. Greilich, A. S. Bracker, and D. Gammon, Nat. Phys. 7, 223 (2011).

${ }^{16} \mathrm{G}$. Muñoz-Matutano, J. Canet-Ferrer, P. Alonso-Gonzalez, B. Alen, I. Fernandez-Martinez, J. Martin-Sanchez, D. Fuster, J. MartinezPastor, Y. Gonzalez, F. Briones, and L. Gonzalez, J. Phys. Conf. Ser. 210, 012028 (2010).

${ }^{17} \mathrm{G}$. Muñoz-Matutano, M. Royo, J. I. Climente, J. Canet-Ferrer, D. Fuster, P. Alonso-González, I. Fernández-Martínez, J. MartínezPastor, Y. González, L. González, F. Briones, and B. Alén, Phys. Rev. B 84, 041308(R) (2011)

${ }^{18}$ X. Zhou, S. Sanwlani, W. Liu, J. H. Lee, Zh. M. Wang, G. Salamo, and M. F. Doty, e-print arXiv:1109.4566v1.

${ }^{19}$ J. Peng, C. Hermannstadter, M. Witzany, M. Heldmaier, L. Wang, S. Kiravittaya, A. Rastelli, O. G. Schmidt, P. Michler, and G. Bester, Phys. Rev. B 81, 205315 (2010).

${ }^{20}$ T. Hatano, M. Stopa, and S. Tarucha, Science 309, 268 (2005).

${ }^{21}$ C. Hermannstadter, G. J. Beirne, M. Witzany, M. Heldmaier, J. Peng, G. Bester, L. Wang, A. Rastelli, O. G. Schmidt, and P. Michler, Phys. Rev. B 82, 085309 (2010).

${ }^{22}$ B. Szafran and F. M. Peeters, Phys. Rev. B 76, 195442 (2007). 
${ }^{23}$ Z. Barticevic, M. Pacheco, C. A. Duque, and L. E. Oliveira, J. Phys. Condens. Matter 21, 405801 (2009).

${ }^{24}$ J. Peng and G. Bester, Phys. Rev. B 82, 235314 (2010).

${ }^{25}$ B. Szafran and F. M. Peeters, Phys. Rev. B 72, 155316 (2005).

${ }^{26}$ A. Harju, S. Siljamäki, and R. M. Nieminen, Phys. Rev. Lett. 88, 226804 (2002).

${ }^{27}$ L. Jacak, P. Hawrylak, and A. Wojs, Quantum Dots (SpringerVerlag, Berlin, 1998).

${ }^{28}$ J. I. Climente, M. Korkusinski, G. Goldoni, and P. Hawrylak, Phys. Rev. B 78, 115323 (2008).

${ }^{29}$ M. F. Doty, J. I. Climente, M. Korkusinski, M. Scheibner, A. S. Bracker, P. Hawrylak, and D. Gammon, Phys. Rev. Lett. 102, 047401 (2009).

${ }^{30}$ C. Y. Hsieh, R. Cheriton, M. Korkusinski, and P. Hawrylak, Phys. Rev. B 80, 235320 (2009).

${ }^{31}$ W. H. Press, S. A. Teukolsky, W. T. Vetterling, and B. P. Flannery, Numerical Recipes in Fortran 77 (Cambridge University, Cambridge, England, 1996), Vol. 1.

${ }^{32}$ [https://code.google.com/p/citool/].
${ }^{33}$ F. Rajadell, J. I. Climente, J. Planelles, and A. Bertoni, J. Phys. Chem. C 113, 11268 (2009).

${ }^{34}$ M. Levinshtein, S. Runyantsev, and M. Shur, Handbook Series on Semiconductor Parameters (World Scientific, Singapore, 1999), Vol. 2.

${ }^{35}$ M. F. Tsai, H. Lin, C. H. Lin, S. D. Lin, S. Y. Wang, M. C. Lo, S. J. Cheng, M. C. Lee, and W. H. Chang, Phys. Rev. Lett. 101, 267402 (2008).

${ }^{36}$ This behavior is exclusive of $X^{+}$, because for indirect $X^{-}$the hole tunneling is negligible.

${ }^{37}$ P. A. Dalgarno, J. M. Smith, J. McFarlane, B. D. Gerardot, K. Karrai, A. Badolato, P. M. Petroff, and R. J. Warburton, Phys. Rev. B 77, 245311 (2008).

${ }^{38}$ J. I. Climente, A. Bertoni, and G. Goldoni, Phys. Rev. B 78, 155316 (2008).

${ }^{39}$ A. V. Koudinov, I. A. Akimov, Yu. G. Kusrayev, and F. Henneberger, Phys. Rev. B 70, 241305(R) (2004).

${ }^{40}$ M. F. Doty, J. I. Climente, A. Greilich, M. Yakes, A. S. Bracker, and D. Gammon, Phys. Rev. B 81, 035308 (2010). 